\title{
La mirada sociológica hacia la alimentación: análisis crítico del desarrollo de la investigación en el campo alimentario
}

\author{
Cecilia DÍAZ MÉNDEZ \\ Departamento de Sociología - Universidad de Oviedo \\ cecilia@uniovi.es \\ Isabel GARCÍA ESPEJO \\ Departamento de Sociología - Universidad de Oviedo \\ igarcia@uniovi.es
}

Recibido: 08-06-2013

Aceptado: 26-02-2014

\section{Resumen}

La Sociología de la Alimentación es un área de trabajo relativamente reciente que sólo en los últimos años ha cobrado relevancia científica internacional como disciplina de estudio (Mennell, Murcotrt y van Otterloo, 1992; McIntosh, 1996; Beardsworth y Keil, 1997; Germov y Williams, 2000; Koc, Sumner y Wilson; 2012). El retraso en la constitución de un cuerpo científico propio es resultado de un conjunto de factores: la diversidad de temáticas existentes, las dificultades metodológicas para afrontar un campo de naturaleza multidimensional o la escasez de estudios comparados explican, al menos parcialmente, su posición académica. Este artículo resume la forma en que la Sociología afronta el estudio de la alimentación. Para ello se ha realizado un estudio de las publicaciones científicas de los últimos 20 años a través de los artículos sobre alimentación publicados en las revistas de Sociología del Journal Citantion Report. Este trabajo permite presentar la panorámica de los estudios de Sociología de la Alimentación en el ámbito internacional y realizar algunas propuestas críticas sobre su desarrollo. Tras este recorrido se puede afirmar que la Sociología de la Alimentación cuenta con un ámbito de estudio propio, aunque con frecuencia se mueva en las fronteras de la Sociología Rural y la Sociología del Consumo.

Palabras clave Sociología de la Alimentación, Sociología del Consumo, Sociología Rural, homogeneización alimentaria, globalización alimentaria, desigualdades alimentarias, sistema agroalimentario

\section{A sociological perception of food: a critical analysis of research develop- ments in the study of food}

\begin{abstract}
The Sociology of Food is a relatively new area of work which only in recent years has gained international scientific relevance as a discipline of study (Mennell, Murcotrt and van Otterloo, 1992; McIntosh, 1996; Beardsworth and Keil, 1997; Germov and Williams, 2000; Koc, Sumner and Wilson, 2012). The delay in the formation of a specific scientific body is the result of a combination of factors: the diversity of subjects, the methodological difficulties to face a multidimensional field of study, or the scarcity of comparative studies. This paper summarizes how sociology undertakes the study of
\end{abstract}


food. We made a study of the scientific articles on food that have been published in the last 20 years in the journals of Sociology that appear in the Journal Citation Report. This paper allows us to present an overview of the studies on the Sociology of Food at the international level and to make some critical proposals as to their evolution. As a result, we may conclude that the Sociology of Food occupies a specific field of study, although it frequently overlaps the fields of Rural Sociology and Sociology of Consumption.

Key words: Sociology of Food; food studies, food consumption, Rural Sociology, food homogenaization, food globalization, food inequalities, agrofood system

\section{Referencia normalizada}

Díaz Méndez, C., García Espejo, I. (2014). “La mirada sociológica hacia la alimentación: análisis crítico del desarrollo de la investigación en el campo alimentario”. Política y Sociedad, Vol.51 Núm. 1 $15-49$

Sumario: 1. El estudio sociológico de la alimentación. 2.Metodología de trabajo: revisión bibliográfica del Journal Citation Report. 3.De la problematicación de la agricultura a la problematización de la alimentación. 4.Desigualdades alimentarias y globalización: de la cultura de clase a la alimentación global. 5.Identidades alimentarias: alimentación, cuerpo y salud. 6.Conclusiones. Bibliografía 


\section{El estudio sociológico de la alimentación}

La Sociología de la Alimentación es un área de trabajo relativamente reciente que, a lo largo de las últimas décadas, ha cobrado relevancia científica internacional como disciplina de estudio (Mennell, Murcott y van Otterloo, 1992; McIntosh, 1996; Beardsworth y Keil, 1997; Germov y Williams, 2000; Koc, Sumner y Wilson; 2012). A pesar del aumento sostenido de publicaciones científicas en las que los sociólogos estudian las diversas y complejas caras de la alimentación en las sociedades modernas, aún se está lejos de la constitución de un cuerpo científico propio. Este retraso es resultado de un conjunto de factores diversos, entre los que se pueden destacar al menos cuatro: En primer lugar, la variedad de temáticas existentes que hacen de este campo un área de gran interés investigador, pero con frecuencia excesivamente abierto y poco delimitado. En segundo lugar, por las dificultades para afrontar estudios comparados que permitan clarificar las hipótesis acerca de si nos encontramos ante rasgos de carácter cultural específicos de un territorio o ante tendencias de cambio social más amplias que puedan ser comprendidas y analizadas como tendencias propias de la modernidad e integradas en teorías sociológicas más amplias sobre el cambio social. En tercer lugar, debido a la dificultad para abordar un área de estudio que por su naturaleza holística requeriría de aproximaciones multidisciplinares que, sin embargo, apenas se realizan. Y en cuarto lugar, debido a la división de las metodologías empleadas que separan de manera sistemática los estudios cuantitativos y cualitativos, como sucede en otros temas de estudio sociológicos, pero aquí resulta más limitador al ser la alimentación un campo multidimensional que requiere, más que otros, de triangulación metodológica.

\section{Metodología de trabajo: revisión bibliográfica del Journal Citation Report}

Una de las formas posibles de conocer el peso científico de un campo de estudio concreto es a través de las publicaciones científicas en ese campo. Hemos tomado como ejemplo el análisis de las publicaciones sociológicas sobre alimentación siguiendo la lógica de describir la disciplina a través de lo que hacen los estudiosos que se dedican a ella. En definitiva, sería considerar que la Sociología de la Alimentación es lo que hacen los sociólogos que se dedican a su estudio, algo seguramente parcial, pero que ayuda a centrar un campo científico que no ha logrado aún una clara delimitación.

Para averiguar cómo ha sido tratada la alimentación desde las Ciencias Sociales se ha realizado un análisis bibliográfico de las publicaciones del Journal Citation Report (Social Science) a través de la Web of Knowledge (WOK) una de las plataformas de recogida de las publicaciones científicas, gestionada en España por la Fundación Española para la Ciencia y la Tecnología (FECYT). El registro de la WOK presenta, sin duda, una perspectiva parcial de la sociología internacional, pues la preponderancia de la lengua inglesa supone una presencia mayor de la Sociología americana y británica en detrimento de los trabajos escritos en otras 
lenguas. Sin embargo permite constatar la evolución de los estudios sociológicos sobre alimentación en el ámbito académico, dada la consolidación del registro como referencia de calidad en las instituciones universitarias internacionales. Se han analizado las 128 revistas de Sociología indexadas y se han explorado en ellas los estudios dedicados a la alimentación a través de las palabras clave food or diet* or eat* or cook ${ }^{* 1}$. Se ha acotado un período temporal de 1990 al 2013, tras comprobar la escasa presencia de artículos anteriores a esta fecha (solamente 4$)^{2}$.

El número de trabajos publicados bajo los epígrafes de búsqueda es de 1.629, de los cuales se diferencian 397 cuando se perfila la búsqueda hacia los estudios que empleen metodologías cualitativas con las palabras qualitative or interview or focus group or case study or life historie. El número de trabajos cuantitativos bajo los epígrafes quantiative or survey or sample or statistics or source or data or regression analysis or model or variable es de 591.

No se pretende una exploración exhaustiva de todas las publicaciones, pero sí abordar un objetivo: analizar los temas tratados y su evolución a lo largo del tiempo para relacionar los cambios y el tratamiento de los temas con las problemáticas alimentarias de cada periodo. La exploración permite contar con una visión panorámica de los estudios sobre alimentación escritos por la Sociología académica internacional. Tras este recorrido estamos en condiciones de analizar la consistencia de un campo de estudio como la Sociología de la Alimentación, así como comentar críticamente la contribución de la Sociología a su creación.

Los estudios de este campo de estudio se iniciaron en los años noventa y tienen su máximo desarrollo al final de la primera década del siglo XXI. A partir de los años noventa se constata un crecimiento sostenido de los trabajos científicos y a finales de la década de los noventa se produce una intensificación de la producción científica y se desarrolla de manera significativa a mediados del 2000. Hablamos, por tanto, de un campo científico relativamente nuevo en la Sociología y que da signos de estabilidad y continuidad en los últimos veinte años.

La evolución y el tratamiento de las temáticas clarifican la situación de este campo académico en la Sociología internacional. Se pueden destacar tres área de estudio claramente diferenciadas y consolidadas: una de ellas con un vínculo más claro en el campo de la producción agroalimentaria, una segunda orientada al análisis de las desigualdades sociales y un tercer grupo de temas, más heterogéneo, orientado hacia el análisis de la identidad y el cuerpo.

\footnotetext{
${ }^{1}$ Ha de tenerse en cuenta que los artículos publicados en idiomas distintos al inglés cuentan con un resumen en este idioma, por lo que aparecen igualmente en el listado.

${ }^{2}$ Con la entrada de empleo y desempleo (employ* or unemploy*) el número de referencias desde 1990 hasta la actualidad es de 9.060 y con el término género (gender) de 10.942.
} 


\section{De la problematicación de la agricultura a la problematización de la alimen- tación}

Los estudios de los años noventa tratan la alimentación desde el ámbito exclusivo de la producción agraria. Estos trabajos tienen como motor de análisis los procesos de cambio y la creciente problematización de una agricultura que se industrializa. Los textos se sitúan en el centro de este proceso de industrialización, reflejando la transformación de una actividad ligada a la tierra, dirigida por pequeños propietarios, que se introduce progresivamente, y podría decirse que con resistencias que les particularizan, en un mercado agroalimentario globalizado.

\subsection{Primeros efectos: cambios en la dieta de los más pobres}

En las primeras décadas las temáticas nutricionales marcan las tendencias de los estudios llevados a cabo en países con un escaso nivel de desarrollo, muy especialmente con estudios apoyados en investigaciones cuantitativas. Estos trabajos analizan las variaciones en el estado nutricional de la población y asocian sus transformaciones a los cambios productivos generados por la mercantilización de producciones locales. Los datos proceden del análisis sobre el estado nutricional de la población, de ahí que aparezcan clasificados como cuantitativos, pero se trata de estudios de caso que son utilizados para explicar cómo la intensificación de la producción agrícola y la exportación hace disminuir el consumo de esos alimentos entre la población que los produce empeorando su dieta.

Es el caso del trabajo desarrollado por Wimberley y Bello (1992) en 59 países del tercer mundo analizando el consumo alimentario y el estado nutricional de la población; o el estudio de caso de las comunidades de pastores etíopes cuyo estado nutricional empeora con el aumento de la venta de leche (Holden, Coppock y Assefa, 1991). Estos análisis continúan hasta el final de la década de los noventa y muestran las estrategias de adaptación de los productores pobres de comunidades tradicionales que se enfrentan a situaciones de escasez alimentaria. Los factores generadores de cambio son diversos y han sido analizados con datos cuantitativos: cambios en los tipos de productos, en las técnicas de producción o en los sistemas de distribución y trasporte. Las dietas empeoran con la intensificación y la industrialización de la agricultura de los más pobres. Algunos ofrecen alternativas al respecto, como generar empleos no ligados a la agricultura para evitar estar sujetos al impacto de sus cambios (Campbell, 1999) otros destacan las ventajas de las formas tradicionales de producción (Smith y Smith, 1999).

Este tipo de trabajos tiene continuidad en la década siguiente. Con la misma lógica, es decir, la de mostrar sociedades pobres que se ven afectadas por los mercados globales, ahora se presenta una nueva situación alimentaria: el deterioro nutricional ligado al sobrepeso y la obesidad cuando una sociedad se inserta en la economía de mercado. Así se analiza el caso de los indígenas de la amazonía a 
través del estudio antropométrico y socioeconómico realizado por Welch et al. (2009).

Este conjunto de estudios agroalimentarios desarrollados en países pobres contribuyen en tres direcciones a la comprensión del cambio social en este período histórico: En primer lugar, se muestra el impacto provocado por la inserción de la agricultura en las lógicas de un mercado alimentario global, con el gran efecto que esto supone sobre formas de producciones más tradicionales y las sociedades en las que se desarrollan. Se detecta el fuerte impacto de la globalización económica sobre la población, a través no solamente en los cambios ligados a las actividades productivas, algo estudiado por la Sociología Rural, sino también sobre la alimentación cotidiana y el consumo. Esta orientación analítica complementa la orientación tradicional realizada desde la Sociología Rural y traslada las lógicas de transformación al campo alimentario y con ello a los individuos afectados. En segundo lugar, aunque algunos estudios plantean propuesta de análisis nuevas o profundizan en las formas de resistencia de las comunidades tradicionales, ponen de manifiesto cómo la globalización alimentaria les imprime unas dinámicas transformadoras sobre las que tienen escaso control y una limitada capacidad de resistencia. En tercer lugar, los estudios permiten una visión de los efectos del cambio sobre la dieta a lo largo del tiempo. Se puede ver cómo el proceso de transición nutricional es un camino que comienza en el hambre hasta llegar a la malnutrición, pero cuyo paso siguiente es el sobrepeso y la obesidad. Ambos, malnutrición y obesidad, son resultado del mismo proceso de modernización social.

\subsection{Cambios en la cadena de comercialización de los alimentos: de la tierra al plato}

Al final de la década de los noventa se entra en una nueva etapa marcada por la creciente complejidad de la cadena agroalimentaria. Los trabajos ponen de manifiesto la impactante trasformación de un sector que pasa de una producción local y tradicional, regentada por pequeños productores y dirigida a un consumidor próximo y un productor conocedor del entorno, a una nueva forma de organización productiva plenamente integrada en nuevas formas de comercialización y que distancia a productores y consumidores e introduce nuevos agentes en la cadena agroalimentaria.

Los análisis se centran en los cambios sufridos en los países desarrollados y analizan en paralelo el mundo de la producción y el del consumo. Aunque reconocen que las transformaciones de la agricultura son consecuencia de los cambios en las demandas de los consumidores y a la inversa, es decir, que las formas de producción imprimen nuevas dinámicas a las demandas de los consumidores.

Sí coinciden en los efectos de la globalización alimentaria, pues detrás de ella se encuentra un consumidor confuso al elegir qué comprar-comer y preocupado por la seguridad y el riesgo de los alimentos. Ambos aspectos están íntimamente ligados al aumento de la distancia entre los productores y los consumidores. Esta distancia ha 
afectado al ámbito de la producción en una disminución de la capacidad de control de las actividades productivas. A lo largo del proceso de industrialización de la agricultura el productor ha ido perdiendo protagonismo y capacidad de decisión, trasladándose el poder hacia los eslabones finales de la cadena y dejando en manos de los canales de distribución alimentaria una buena parte de las decisiones sobre qué, cómo y para quién producir. Pero además, el distanciamiento entre productor y consumidor supone un desconocimiento de cómo se producen los alimentos, y esto ha derivado en una preocupación creciente por los riesgos que entrañan los alimentos y un interés manifiesto por comer saludablemente.

El título del trabajo de Buck et al. From farm to table (1997), repetido posteriormente en múltiples estudios, muestra la necesidad de afrontar el análisis del recorrido de los alimentos "de la tierra a la mesa" que requiere una mirada holística para su comprensión. En este trabajo se analizan, mediante un gran número de entrevistas semiestructuradas, los discursos de los actores principales de la cadena de comercialización de productos orgánicos vegetales en California (productores, manipuladores, minoristas, procesadores, inversores, agencias de certificación y académicos). Exploran las dinámicas internas de las interacciones y las relaciones más relevantes. Detectan y analizan las trasformaciones ofreciendo ejemplos de cómo algunas partes de la cadena de comercialización, sobre todo las que tienen mayor valor añadido, han cambiado de manos y han hecho derivar las formas tradicionales de organizar la cadena hacia formas de organización industriales.

Se puede considerar el fin de la década de los noventa como la puerta de entrada de la alimentación en la nueva etapa de modernidad social. Parafraseando el título del trabajo de Shaw What are they doing to our food? (1999), la pregunta pone de manifiesto la incertidumbre reinante en torno a la alimentación en las sociedades desarrolladas. El texto, resultado de una investigación basada en entrevistas en profundidad a informantes clave, refleja el paso de los problemas alimentarios a la esfera pública en un país, Reino Unido, que podría representar al resto de las sociedades desarrolladas del momento. A través del discurso de los expertos entrevistados se repasan los temas alimentarios más problemáticos en un contexto de crisis alimentaria y se presentan en paralelo al aumento de la percepción de los riesgos por parte de la sociedad británica. El ciudadano comienza a cuestionar la forma en que llegan a su mesa los alimentos. Las temáticas reflejan sus preocupaciones, muy especialmente los asuntos asociados a la seguridad y el riesgo alimentario como reflejo de la desconfianza en la alimentación y en sus agentes.

El tema de la seguridad alimentaria comienza antes de la primera crisis global de las vacas locas. Tendrá un amplio desarrollo a partir de este suceso, pero ya en los años noventa se plantea que la percepción del riesgo está condicionada por factores de tipo social. Con análisis basados en pruebas de consenso cultural, Johnson y Griffith (1996) afirman que los consumidores perciben importantes riesgos en pescados y mariscos debido a su percepción acerca de la contaminación del mar. En 1998 en Noruega (Nygard y Storstad, 1998) se debate si los consumidores consideran más seguros los productos nacionales-regionales-locales, con una interesante reflexión de los autores acerca de si estas percepciones y otros aspectos ligados a la 
tradición alimentaria nacional pueden ser un freno o un acicate a la comercialización. El etiquetado de productos locales puede ser una vía de resistencia a la entrada de productos extranjeros más baratos a través de la cual se unen los intereses de productores y consumidores. Lo cercano es más confiable y seguro. Entra en juego una nueva racionalización sobre los alimentos en la que el precio no es el único ni principal criterio de elección y los productos locales pueden garantizar la seguridad y la calidad en un contexto alimentario confuso.

Los estudios se hacen eco del cambio de valores hacia la alimentación en los países desarrollados. Estos cambios no tienen que ver sólo y exclusivamente con el producto, sino con lo que le rodea. En 1995 aparece el primer trabajo cuantitativo sobre vegetarianismo en el que Dietz et al. exploran a través de una encuesta a una muestra de población en Estados Unidos, los factores que influyen en la elección de esta dieta. Parece que los valores altruistas están ligados a la elección, como también confirma posteriormente Kalof et al. en 1999, aunque el predictor más consistente es la creencia de que estas dietas son un beneficio para la salud y el medio ambiente.

También los análisis sobre el turismo se adentran en el estudio de los valores analizando la alimentación como reclamo turístico, aunque con focos de atención diferenciados. En unos los mercados locales se presentan como continuidad de la granja (Miele y Murdoch, 2002), los restaurantes y cocineros como medio de proyección de la comida y los productos locales (Starr et al., 2003; Inwood et al., 2009) o el agroturismo como práctica sostenible (Torres, 2002; Che et al., 2005). Se liga la promoción turística de la comida a los valores sociales en alza de un nuevo "consumidor de territorio" para el que la comida constituye un elemento cultural simbólico.

Los trabajos sobre el bienestar animal (Hyers, 2006; Heleski et al., 2006; Pallotta, 2008; Deemer y Lobao, 2011) reflejan también las nuevas preocupaciones e inquietudes despertadas en torno al sistema agroalimentario, en este caso por la situación de los animales en las granjas, por las actitudes que la población tiene hacia sus condiciones y por las atribuciones de responsabilidad ante el maltrato animal. Algunos autores elaboran tipologías de actitudes y creencias clasificando a la población, como realiza Vinnari et al., (2013) a partir de una amplia muestra de finlandeses.

Realmente no se sabe si las preocupaciones sobre alimentación, las percepciones del riesgo o los nuevos valores ligados al consumo difieren por países, pero se intuye que pueden darse diferencias entre países ligadas a la cultura alimentaria de una sociedad. El tema vuelve a aparecer en un estudio comparativo sobre la confianza en la seguridad de los alimentos realizado en Rusia, Dinamarca y Noruega por Berg et al. (2005) a través de una encuesta a consumidores. Se perciben diferencias entre países en relación a la confianza, pues los consumidores en los países escandinavos sustentan su confianza en el estado mientras que los rusos se la otorgan a los mecanismos reguladores del propio mercado. Esta diferencia en la confianza del consumidor en función del papel del estado se detecta también en un estudio comparado a través de encuestas en Estados Unidos, Reino Unido, Dina- 
marca y Suecia realizado por Sonderskov y Daugbjerg (2010) al estudiar las respuestas al etiquetaje ecológico. Se podría concluir que el consumidor es más confiado en los países en los que el estado está más implicado en la regulación del sistema agroalimentario.

Los estudios comparados muestran la necesidad de considerar las condiciones institucionales específicas a nivel nacional cuando se estudian cuestiones de consumo alimentario. La comparación permite comprender las respuestas de los ciudadanos a la seguridad alimentaría y su grado de confianza, y es una orientación necesaria para cualquier tema que deba ser regulado a nivel nacional, aún existiendo legislaciones supranacionales al respecto.

Todos estos estudios, aunque pretenden establecer vínculos entre la demanda de un consumo más responsable y crítico y la oferta alimentaria, no ofrecen conexiones que permitan constatar el grado de influencia del consumidor sobre la cadena de producción. Muestran que cambian los valores, pues reflejan nuevas preocupaciones acerca de cómo se produce pero, en general, presentan un consumidor ajeno al resto del sistema con nula capacidad de influencia, aunque con frecuencia sean sus respuestas lo que se estudia. Sin embargo, algunos de los análisis sociológicos hacen de los consumidores los últimos responsables del replanteamiento analítico al que se ven abocados los estudiosos del mundo agrario. Los nuevos valores están ejerciendo una fuerte influencia en las formas de producir y con ello alterando la manera de enfrentar el trabajo agrario y ganadero. En definitiva, la preocupación acerca de los cambios alimentarios "entra”, en esta década, a través del consumidor reformulando las orientaciones de los analistas y dando un mayor protagonismo a quienes se ocupan del consumo y dejando en un segundo plano y en otro campo académico a la Sociología Rural, quienes se ciñen al estudio de los aspectos exclusivamente productivos de los alimentos.

\subsection{Convencionalización o resistencia de la agricultura tradicional}

Los textos que predominan en los inicios del siglo XXI están relacionados con un nuevo papel del agricultor en las sociedades desarrolladas. Abundan los trabajos relacionados con el rol que los pequeños agricultores tienen en el desarrollo rural de áreas geográficas concretas. Se trata de trabajos en los que se pone de manifiesto la forma en que los pequeños agricultores de los países ricos se han adaptado (y sobrevivido) ya no a la industrialización, sino a los cambios generados por la globalización del sistema agroalimentario. Basándose en la idea de la resistencia del pequeño agricultor, estos trabajos pretenden revitalizar su papel, pero ahora ya no como mero productor de alimentos, sino como cuidador del espacio rural. Ponen en valor el rol del pequeño productor como nexo entre la naturaleza y la sociedad, entre la producción y el consumo, manifestando con ello la función social que éste adquiere a través de la revitalización del espacio rural y reclamando un mayor protagonismo en la relación con el consumidor. 
El elemento común y el debate teórico de fondo es el proceso de "conventionalization”, termino inexistente en castellano que hace referencia a la inserción de la agricultura en las normas de funcionamiento de la economía de mercado capitalista. Algunos autores juegan con la dualidad convencional/alternativo, para otros se trata de una contraposición entre industrial/tradicional. En cualquier caso el foco de atención de los analistas ha cambiado. No se estudia ya la estrategia de resistencia minoritaria de productores marginales y/o alternativos ligados al ámbito de la producción, sino las estrategias alternativas de producción que encuentran una respuesta de consumo tan favorable como para conformarse como alternativas a las cadenas convencionales de producción, distribución y consumo. Es justamente este aspecto lo que vincula productores y consumidores. Se analizan las pautas de cambio seguidas por los productores y se habla de ellas como alternativas capaces de mantenerse en un mercado agroalimentario global con formas no convencionales y sin embargo no marginales. Con esta lógica de fondo, los analistas buscan conocer si las producciones no convencionales, como los productos orgánicos, se convencionalizan o si se desarrollan como formas alternativas de relación entre productores y consumidores.

El título del trabajo de Murdoch y Miele (1999) Back to nature. Changing "worlds of production" in the food sector, muestra al menos dos mundos productivos en el nuevo escenario alimentario global, y no solamente uno. A través de dos estudios de caso italianos en el texto se confirma la coexistencia de formas de producción diferentes frente a la apariencia de homogeneización propiciada por la industrialización de la alimentación. Los autores sostienen que ha tenido lugar una fragmentación de los procesos productivos y en el caso de la alimentación coexisten formas alternativas y convencionales, estas últimas marginan a la naturaleza en sus pautas productivas pero otras tienen un respaldo en un nuevo consumidor preocupado por la seguridad y la salud que otorga nuevos valores a la producción no estandarizada.

Uno de los trabajos más citados por los sociólogos para mostrar estos cambios es el de Marsden et al. (2000) con el título Food supply chain approaches: exploring their role in rural development. En este trabajo se analiza el papel de las cadenas cortas de comercialización como impulsoras del desarrollo rural y es un trabajo que ha servido de base para multitud de estudios empíricos de todo el mundo. Los autores explican, a través de un estudio de caso de la producción de ternera en Gales, el papel de las cadenas cortas de distribución. Ofrecen una tipología de varios niveles y diferentes tipos de evolución de estas cadenas y analizan sus potencialidades considerando cómo hacer frente a las grandes industrias a través de los vínculos entre productor y consumidor y con el apoyo de las instituciones. Este análisis se inserta en la crítica a la creciente complejidad de la cadena agroalimentaria que ha aumentado la desconfianza en la alimentación. Los canales cortos de comercialización pueden ser la estrategia para reducir la distancia entre productor y consumidor y generar confianza. La tipología en tres niveles que plantean constituye una de las propuestas teóricas más interesantes de este campo de estudio. En general, estos trabajos presentan situaciones positivas de trasformación de la agri- 
cultura tradicional que se abre, no sin dificultad, al mercado. Otros ponen el acento en la dificultad que entraña la adaptación de las formas de producción minoritarias al competitivo mercado global. Así, Hall y Mogyorody (2001) estudian también las estrategias seguidas por los agricultores canadienses. A través de una encuesta a una muestra de granjas ecológicas que tienen una mayor orientación hacia la producción de orgánicos confirman que conservan los rasgos de un sistema alternativo de producción, incluyendo la venta directa al consumidor. Postulan que se mantienen sus estrategias de producción alternativas y no se convencionalizan.

Estas posiciones se complementan con los análisis en los que no se detecta tanto un mundo dividido como estrategias diferentes en función del contexto en el que se producen. Así se ve en el estudio comparado sobre las redes de comercio justo analizadas por Garcia y Lara (2004) entre Estados Unidos y México. Analizando varias experiencias en ambos países, confirman que si para los primeros el comercio justo tiene relevancia como forma de preservación de la agricultura familiar, para los mejicanos se trata más de estrategias de entrada en el mercado a través de certificaciones de origen. Esta cuestión hace reflexionar acerca de la necesidad de vincular las explicaciones sobre el desarrollo de estas redes alternativas al territorio en el que nacen y no solo al consumidor al que llegan. El título del trabajo de estos autores Bringing the moral change home: fair trade within the north and whiting the south expresa bien la dualidad moral existente en torno al valor de justicia social de los países ricos y pobres. El debate no está tanto entre si se convencionalizan o no las formas alternativas de relación entre productores y consumidores, sino si realmente los productores pueden mantener estrategias no convencionales en un mercado global. Los países ricos tienen ejemplos que confirman esta posibilidad, pero no es tan evidente entre los países pobres, que se muestran más preocupados por sobrevivir, sea cuál sea la fórmula, que por mantener pautas alternativas de acción que den respuesta a un consumidor más exigente. La convencionalización es una estrategia inevitable para unos mientras que para otros es una opción.

\subsection{El debate sobre la convencionalización a través del análisis de los actores en la cadena agroalimentaria}

Aunque las investigaciones muestran datos contradictorios al respecto, se ha desarrollado una importante línea de estudio que ha pretendido vincular analíticamente al productor y al consumidor intentado explicar el proceso a través del cual se hace más compleja la cadena agroalimentaria. Teniendo en cuenta que esta complejidad corre en paralelo al aumento de los actores intervinientes entre el productor y el consumidor, algunos analistas se han adentrado en el estudio de los roles y el poder de estos agentes, de ahí ha surgido una de las orientaciones más fructíferas. Un ejemplo de ello es el desarrollo de la teoría del actor red (Actor Network Theory) aplicada a las relaciones entre naturaleza y sociedad que pone énfasis en las relaciones de poder en la cadena agroalimentaria. Lo ha planteado por primera vez Goodman (1999) en Agro-food studies in the "age of ecology". Nature, corporeality, bio- 
politics. La autora analiza las relaciones entre productor y consumidor en el caso de la agricultura ecológica australiana y explica cómo se producen vínculos basados en una ética inherente a las relaciones que se dan en este tipo de agricultura. Campbell y Liepins (2001), en Nueva Zelanda, argumentan en la misma línea en uno de los trabajos más citados hasta la fecha. Los autores realizan un recorrido histórico por el proceso de transformación de la agricultura y muestran cómo van cambiando las ideas sobre los productos orgánicos a lo largo del tiempo. Al realizar un análisis de los cambios desde los años setenta se comprenden bien las variaciones en las posiciones de poder en la cadena agroalimentaria y se constata el desplazamiento de poder hacia los agentes más próximos al consumidor a la vez que los productores van perdiendo protagonismo.

También indaga sobre los vínculos productor-consumidor apostando por nuevas formas de relación alternativas el trabajo de Raynolds (2002) titulado Consumer/producer links in Fair Trade coffee networks. La autora argumenta que los elementos de unión se sustentan en la justicia social, la confianza y la equidad. Estos mercados alternativos abren la posibilidad de desarrollar relaciones nuevas en la producción, el comercio y el consumo. El éxito del mercado de café dentro de las redes de Comercio Justo es un ejemplo, pues desafía a los mercados convencionales dominados por las grandes corporaciones alimentarias. Este tipo de redes hacen más próxima y más igualitaria la relación entre productor y consumidor y, además, humanizan el proceso de intercambio comercial.

Profundiza en estos análisis en las sociedades occidentales Goodman (2004) Rural Europe redux? Reflections on alternative agrofood networks and paradigm change. Analiza otras redes agroalimentarias alternativas planteando que también en Europa hay vínculos nuevos entre productores y consumidores que podrían ser formas alternativas de relaciones en el sistema agroalimentario vinculadas al desarrollo rural.

Tanto Raynolds, Goodman, como Campbell y Liepins (2001) abren una vía de análisis muy prolífica en la segunda mitad del 2000 al plantear la posibilidad de explicar el funcionamiento del sistema agroalimentario a través de las relaciones entre los actores que intervienen en ella. También introducen una crítica sobre la perspectiva determinista con la que se había planteado el análisis de la transformación del sistema agroalimentario mundial hasta el momento.

Pero también hay autores que no se muestran tan convencidos de que las redes alternativas de comercialización se mantengan realmente como alternativas y no como pautas marginales de relación en el sistema agroalimentario. El trabajo de Lockie et al. (2002) titulado Eating green. Motivations behind organic food consumption in Australia analiza el creciente interés de los consumidores por los alimentos producidos sin agentes químicos, en el propio territorio, con bajo impacto ambiental y sin organismos genéticamente modificados. El trabajo se hace eco de las dificultades de la producción y la industria para dar respuesta a una demanda creciente de los consumidores por los productos orgánicos. A través de grupos de discusión y de una encuesta nacional, los autores examinan el significado de "producto verde” y cómo estos significados se reflejan en las prácticas de consumo. 
Estos análisis introducen un elemento más en el análisis de los cambios en el sistema agroalimentario y sus redes de relación. En ellos, la figura del consumidor y sus preferencias alimentarias es el centro del análisis y se estudia la valoración y los significados que atribuyen a los alimentos para explicar los hábitos de compra y consumo. Sin embargo, no parece que sea fácil determinar cuales son estos valores y cómo inciden, pues los consumidores cuentan con mucha información variada que les orienta en sus elecciones y con criterios diversos que no siempre tienen que ver con una posición ideológica más ligada a los productos alternativos. Criterios como el precio tiene un peso similar a valores como la protección del medio ambiente, y la conveniencia favorecerá el consumo sin contar con cuestiones de carácter ético o ideológico.

Más adelante, en 2005, también Lockie y Halpin (2005) demuestran, con una extensa encuesta a agricultores australianos, que la agricultura no se fragmenta entre alternativos y convencionales. Estudiando a productores grandes y pequeños dedicados a la producción de orgánicos constatan que los valores de unos y otros son coincidentes y los grandes no son los más reacios a apoyar los productos locales. Cuestionan la tesis de la convencionalización, pues no hay muestras de estar ante dos tipo de agriculturas diferenciadas, ni ideológica ni estructuralmente, y los autores afirman que se trata más de una división entre buenos (alternativos) y malos (convencionales) que una correcta orientación para analizar los cambios en las formas de producción. Por lo tanto, dicen, ni estamos ante agriculturas alternativas, ni ante consumidores alternativos.

También el debate sobre la convencionalización de la agricultura se afronta de mejor modo a través de estudios comparados. Es un buen ejemplo de ello el trabajo de Fonte (2008) en el que se realizan estudios de caso en 10 países europeos para conocer el proceso de valorización de los alimentos y analizar la interacción entre las formas de conocimiento y los actores en la definición de la comida local. El estudio se inserta en el debate acerca de si la relocalización de la alimentación es una estrategia que tiene continuidad o no en un mercado alimentario global, en definitiva, si resiste o si sucumbe. Para la autora no existe tal dicotomía, pues depende del contexto en el que se produce la relocalización. En Europa se apuesta políticamente por incorporar al desarrollo económico a los pequeños productores agrarios y la literatura que analiza esta situación incorpora a los consumidores como parte del proceso, de modo que se entiende como algo que es construido por muchos actores en la red agroalimentaria. Al analizar las formas de conocimiento (de los rurales y de los urbanos, de los científicos y de los legos) la autora contribuye a explicar las asimetrías de poder en la interacción. Plantea que para decidir acerca de la persistencia o desaparición de las formas alternativas de producción y consumo es necesario situarse en el contexto en el que se producen y explicar cómo se adaptan a él. Las diferentes situaciones son una muestra de que no hay una única estrategia para afrontar los problemas locales, pues cada territorio tiene sus propias fortalezas y debilidades aunque el objetivo sea en todos los casos lograr un desarrollo rural sostenible. 


\subsection{Cambios en el papel de las instituciones en la cadena agroalimentaria}

No se puede explicar la respuesta ante las presiones de la globalización alimentaria sin el análisis del poder de los agentes que intervienen en la cadena. Los agentes institucionales, en particular el Estado, cobran especial protagonismo adoptando al menos, dos papeles; bien como orientador de las decisiones de los productores, bien como defensor de los intereses de los consumidores. Así, se constituyen en freno o acicate de la actividad productiva.

Trabajos como el de Dupuis (1993) analizando los cambios históricos seguidos en el proceso de reordenación de la industria láctea en Estados Unidos, muestran el papel de los Estados en la ordenación de las políticas agrarias. No es el único trabajo que refleja el papel y el poder de las instituciones sobre los agricultores. El texto de Bonanno et al. (1995) analiza, a través de un estudio de caso italiano, las relaciones entre los Estados y las empresas agroalimentarias y los efectos de estas alianzas sobre los pequeños productores. Aunque los autores muestran un caso en el que los grupos subordinados quedan en peor situación debido a las alianzas entre los agentes más poderosos, el trabajo pone de manifiesto la capacidad para controlar las relaciones y sus efectos por parte de los Estados-nación, un análisis que abre puertas para comprender el papel de las instituciones en entornos diferentes.

Estos trabajos se desarrollan con más intensidad a partir de la segunda mitad de la década del 2000. Uno de los más relevantes en este campo es el de Guthman et al. (2006). Muestra el importante papel de las instituciones en Estados Unidos para vincular los intereses de productores y consumidores. Afirman que el objetivo de lograr alimentos seguros es un asunto importante para ambos, aunque las orientaciones de los productores a favor de la seguridad del consumidor varían según la intervención institucional (ayudas gubernamentales). Este papel intermediador de las instituciones también se pone de manifiesto en los trabajos de Bonanno y Constance (2006) explorando la capacidad del Estado para controlar o regular el gran poder que han ido adquiriendo las empresas agroalimentarias. A través del estudio de los documentos de las operaciones realizadas por las empresas porcinas de Texas, los autores van mostrando las interrelaciones entre ambos y las mutuas interdependencias. Las producciones se convencionalizan si existe un respaldo institucional hacia esas orientaciones.

Tomlinson (2008), a través del estudio de documentos del gobierno y de entrevistas a actores políticos y a agentes de los grupos de productores orgánicos, presenta el relevante papel del gobierno de Reino Unido para el desarrollo de estos productos. El autor explica la forma en que el gobierno ha contenido y regulado la transformación de la agricultura orgánica y de este modo incorpora un actor más en la comprensión del proceso de convencionalización de la agricultura, aunque esta intervención no sea tanto dirigida al desarrollo de la agricultura orgánica como al desarrollo de una alimentación saludable.

Holm y Halkier exploran el proceso seguido por las instituciones para elaborar normas de seguridad alimentaria a escala europea tras la encefalopatía espongiforme bobina, la EEB (2009). Explorando el proceso en un estudio comparativo en seis 
países (Dinamarca, Alemania, Italia, Noruega, Portugal y Reino Unido) concluye que se generaliza un marco normativo con el objetivo de restablecer la confianza del consumidor, pero la aplicación de la norma varía en los diferentes contextos analizados. En definitiva, se converge en la norma pero no en la aplicación. Es posible que esta sea la conclusión de los estudios sobre el rol de las instituciones en el sistema agroalimentario. Ni siquiera ante una norma internacional compartida podemos situarnos en escenarios similares, pues las instituciones nacionales imprimen particularismos a las naciones en su aplicación. Con estas conclusiones se podría pensar que la influencia de las grandes instituciones supranacionales, muy especialmente en el mercado agroalimentario, no genera el esperado efecto homogeneizador en las prácticas políticas.

\subsection{El papel de los grupos de acción}

Es necesario complementar estos análisis de las instituciones formales con la de los grupos de acción que, siendo menos organizados y con un grado de influencia menos visible, introducen variaciones en la cadena agroalimentaria en nombre del consumidor o del pequeño productor. Se pueden enmarcar estos trabajos en la categoría de activismo alimentario y han sido desarrollados por varios autores. Se trata de artículos que exploran iniciativas de acción política en torno a la comida que suponen nuevas relaciones sociales alrededor del sistema alimentario.

El título del trabajo de Roff en 2007 lo expresa de modo muy ilustrativo Shopping for change? planteando las consecuencias negativas para la sociedad de algunos tipos de activismo anónimo e individual al analizar las respuestas de los grupos contra los alimentos genéticamente modificados. Esta autora sostiene que los movimientos activistas se han convertido en movimientos neoliberalizados. Su visión crítica le hace analizar el poder de los consumidores para alterar las prácticas de los fabricantes de alimentos, pero también para ejercer una influencia que se escapa a la representación de la ciudadanía. La idea que subyace es la de la walmartization de la alimentación, comida barata pero social y económicamente cara, que no resuelve los problemas alimentarios actuales y además genera injusticia social.

Con una postura menos crítica a esta forma de participación, el trabajo de Alkon y Norgaard (2009) Breaking the food chains muestra el poder de grupos minoritarios pero relevantes. Las autoras analizan las demandas de dos grupos de activistas americanos que reclaman alimentación saludable, accesibles y culturalmente apropiadas. En la misma línea Brunoni et al. (2012) exploran, con varios estudios de caso, los grupos de compra solidaria y, enmarcando el trabajo en las teorías del actor red, muestran la capacidad de estos grupos para modificar las formas dominantes de gobernanza alimentaria.

Estos trabajos coinciden al centrar el análisis en el grado de influencia de las instituciones públicas, de las empresas o de los grupos de acción para generar transformaciones que afectan de manera decisiva a la cadena agroalimentaria incidiendo en la dirección de los cambios. Como motores de cambio la intervención de estos 
agentes es apropiada para unos e inadecuada para otros, en tanto en cuanto pueden ser (y de hecho son) formas de poder en un sistema agroalimentario que ha aumentado el número de agentes de la cadena agroalimentaria, y, dentro de la cual, no todos ellos tienen la misma capacidad de influencia.

\section{Desigualdades alimentarias y globalización: de la cultura de clase a la ali- mentación global}

En los años noventa se comienza a reflejar, tímidamente, la preocupación por los efectos del cambio alimentario dentro de los hogares, con una orientación basada más en la Sociología del Consumo y al margen de la Sociología Rural. Se trata de estudios que discurren en paralelo a los anteriores a partir de los años noventa pero separados del ámbito productivo. En ellos las aproximaciones cualitativas se acercan al consumidor, y en ocasiones al comensal, y buscan la comprensión de las conductas alimentarias a través de los discursos de los sujetos y de sus percepciones sobre la alimentación y el alimento. Los estudios cuantitativos, por su parte, analizan las conductas más visibles de compra y consumo para explorar las motivaciones de las elecciones de los alimentos y los factores sociales que las explican.

Pero posiblemente lo que caracteriza estos trabajos que se alejan del ámbito productivo sea su gran diversidad temática. Esto tiene dos explicaciones: Por un lado, es posible que estemos ante trabajos que carecen de un cuerpo teórico compartido. Esto dificulta la clasificación y la comprensión de los estudios empíricos como parte de un mismo asunto analítico y la diversidad refleja esta falta de elementos teóricos comunes entre los investigadores que permitan una cierta coherencia y continuidad. Por otro lado, es posible que la diversidad esté reflejando la profusión de perspectivas analíticas sobre las cuales se puede estudiar la alimentación y sus problemas en las sociedades contemporáneas. Sin un soporte común similar al ofrecido por la Sociología Rural a los analistas del mundo productivo, es lógico encontrar estudios que no avanzan en la misma dirección, dado que no son resultado ni de las mismas preguntas, ni de las mismas orientaciones teóricas de análisis.

Otro de los temas sobre los que pivotan los trabajos y que se desarrollan a mediados de la década del 2000, es el estudio de los cambios alimentarios dentro de los hogares. Los cambios sociales generan preocupaciones en la sociedad derivadas especialmente de la perdida de formas de alimentarse ligadas a las culturas nacionales y a la tradición. Se ve con preocupación la perdida de la comida familiar, por el componente de convivencialidad que comporta, y la desaparición de pautas alimentarias culturalmente asentadas que se enfrentan a un proceso homogeneizador derivado de la globalización. Los analistas exploran los patrones alimentarios en los hogares, unos derivan el análisis hacia la comida familiar y su capacidad de resistencia en unas sociedades crecientemente más homogéneas.

A pesar de la diversidad temática reinante, si podríamos decir que el trasfondo analítico sobre el que se asientan los estudios del campo del consumidor es, nuevamente, los efectos que la globalización genera en sus prácticas alimentarias, en 
definitiva, reflexionan acerca de la estandarización que provoca la globalización en los hábitos alimentarios. Bien es cierto que en este caso los aspectos económicos de la globalización quedan en un segundo plano para dejar paso a la preocupación por sus efectos culturales. Las pautas alimentarias muestran signos de homogeneización en una sociedad culturalmente más conectada en la que los modelos alimentarios dominantes, los occidentales, se ven reforzados por la internacionalización de los mercados. Los modelos alimentarios dominantes ejercen una fuerte influencia en las culturas nacionales dando lugar a la preocupación acerca de su resistencia o su desaparición. El debate es similar al anterior, pero si antes la preocupación se centraba en las pautas productivas, ahora preocupa en qué medida la globalización alimentaria genera efectos homogeneizadores sobre unos hábitos alimentarios con fuertes raíces sociales y culturales y de qué modo encuentra resistencias entre los comensales y consumidores.

\subsection{Primeros efectos: nuevas formas de desigualdad en sociedades de abun- dancia.}

Una de las orientaciones que mantiene una cierta coherencia teórica y continuista con la Sociología de otras épocas y otros estudios, es la que se inserta en el análisis de las desigualdades sociales. Esta es una orientación característica de la Sociología que sirve de soporte a los temas alimentarios desde los estudios de malnutrición en los períodos iniciales de la transición nutricional. Las desigualdades continúan siendo una temática de estudio sociológico a pesar de la abundancia que domina el panorama alimentario de la mayoría de los países analizados por la Sociología. Al igual que en los tiempos de escasez, la privación material de los hogares altera su alimentación. Son varios los trabajos que muestran las situaciones de los hogares que reciben ayudas alimentarias en Estados Unidos, un asunto que comienza a verse en los inicios de los noventa en Edin (1991) quien analiza, a través de entrevistas en profundidad, las formas en que las mujeres con hijos de Chicago afrontan la privación material con ayudas gubernamentales. Más recientemente Krueger et al. (2004) se preguntan To help or to harm? poniendo en cuestión si estas ayudas generan dependencia del Estado entre quienes las reciben. Confirma que estas ayudas no son suficientes para afrontar sus situaciones de necesidad y las preceptoras completan estos recursos con otras fuentes de ingresos, como ayudas de familiares o actividades laborales no declaradas.

El tema deja de estar presente en la literatura sociológica hasta mediados de la década del 2000. Siguiendo esta estela e intentando vislumbrar más allá de las ayudas estatales, autores como Krueger et al. (2004) analizan los efectos de estas ayudas sobre la mortalidad. La crisis económica hace a los analistas explorar, ya no solo las formas de afrontar la penuria económica con ayudas gubernamentales, sino las estrategias que los propios hogares elaboran para enfrentarse al hambre. Resultan muy ilustrativos dos estudios, uno en una ciudad de Filipinas (Schmeer, 2005), 
otro en Estados Unidos (Kenney, 2008), que muestran cómo en los hogares en los que las mujeres casadas gestionan los ingresos de forma exclusiva y por tanto se ocupan de las compras de alimentos, sus hijos muestran mejores niveles nutricionales, pues el hogar destina mayores recursos a la alimentación.

Parece claro que el hambre y la malnutrición son problemas que no han desaparecido en las sociedades sobrealimentadas y los analistas asocian estas situaciones a contextos que profundizan en las desigualdades aunque con una nueva cara. Podríamos decir que la obesidad es la manifestación moderna de la pobreza. Con metodología mixta Smith et al., (2010) muestran que un porcentaje elevado de personas sin hogar en Estados Unidos tienen obesidad, muy especialmente cuando residen en zonas de poco acceso a la alimentación fresca. Tanto es así que se habla de desiertos alimentarios para explicar las dificultades de acceso a alimentos saludables entre la población rural y/o más pobre. La escasez de frutas y verduras en las dietas de las familias de bajos ingresos está íntimamente ligada a las oportunidades de acceso a este tipo de productos, como confirman en Estados Unidos varios analistas (Morton et al., 2005; Hendrickson et al., 2006). Aunque no se trata de un tema nuevo en los estudios sobre alimentación, pues es un asunto iniciado en los años noventa en Reino Unido, pone de manifiesto la importante relación entre la salud y la alimentación y cómo ésta se ve afectada de manera significativa en las familias en peores condiciones socioeconómicas. Algunos estudios establecen un vínculo entre la obesidad y la ruralidad en Estados Unidos (Schafft et al., 2009), pero las dificultades en el acceso a productos frescos no es un asunto sólo norteamericano, también genera estratificación social en otros países desarrollados como Japón (Kobayashi, 2010). En definitiva, la desigualdad alimentaria sigue siendo un tema de interés para los sociólogos aunque ahora la pobreza alimentaria ha cambiado de perfil y presenta nuevas situaciones ligadas a las sociedades donde no faltan alimentos. La obesidad es la nueva cara de la pobreza en los países ricos.

Hay un pequeño grupo de estudios que analizan un comportamiento nuevo. Se trata de análisis sobre comportamientos tradicionales que tienen, a buen seguro, una lógica distinta en la actualidad y que los analistas pretenden desentrañar. Es el caso de los análisis realizados sobre la agricultura urbana. Los huertos urbanos han sido analizados para ver si esta práctica es una estrategia para afrontar situaciones de escasez de recursos. Lo han estudiado a través de encuestas oficiales en ciudades rusas (Clarke et al., 2000) y no se puede decir que la agricultura urbana sea una respuesta para afrontar situaciones de crisis. Los hogares rusos que tienen huertos urbanos no son los de más bajos ingresos. Se trata más de un complemento al empleo remunerado que una alternativa ante situaciones de desempleo. A conclusiones similares llegan los estudios realizados en Canadá (Kortright y Wakefield, 2011) que con entrevistas en profundidad demuestran que esta producción de alimentos contribuye a una dieta más saludable en todos los grupos sociales, independientemente de sus ingresos. También se confirman las motivación de salud y de ocio en todos los grupos que tienen huertos en la población checa (Jehlicka et al., 2013). Es evidente, también aquí, que el efecto de la globalización alimentaria sobre los hábitos alimentarios tiene nuevas manifestaciones y que no se puede buscar en 
los mismos lugares que años atrás: ni la penuria económica se manifiesta a través de la infraalimentación, ni las estrategias de subsistencia coinciden con las del pasado.

\subsection{Cambios en los patrones alimentarios: entre la clase social y la individuali- zación alimentaria}

Otro de los asuntos que impregna una buena parte de la literatura sociológica hace referencia a la pervivencia de patrones alimentarios ligados al origen social de los individuos. Este asunto aparece en la literatura sociológica para explicar la menor diversidad alimentaria en las sociedades modernas. La globalización imprime pautas de cambio que unifican los hábitos y ofrece una homogeneidad alimentaria resultado de múltiples factores, desde la internacionalización de los mercados alimentarios hasta la multiculturalidad de las sociedades actuales. Si en las épocas precedentes los gustos aprendidos y reproducidos en las clases sociales mostraban la diversidad social, ahora este efecto de clase se minimiza generando unas pautas alimentarias cada vez más homogéneas. El debate se ha desarrollado en torno a dos aspectos de estas pautas culturales: por un lado, el mantenimiento de las pautas alimentarias ligadas a la clase social; por otro lado, la pervivencia de hábitos alimentarios fuertemente anclados en la cultura, como la comida familiar.

La apariencia de comportamientos alimentarios más homogéneos entre la población puede estar indicando que las desigualdades se han aminorado, aunque también puede ser reflejo de una disminución de las pautas alimentarías nacionales que tienden a parecerse en una sociedad que socava la diversidad cultural a favor de comportamientos culturalmente más homogéneos. El debate sobre la desaparición de las clases sociales y la homogeneización alimentaria de las sociedades modernas confluye y se desarrolla también en relación a la transformación de las pautas alimentarias tradicionales. Si los modelos alimentarios basados en el origen social mantienen sus especificidades estamos ante hábitos alimentarios que se reproducen y se ligan a la clase social. Si por el contrario se muestran pautas similares entre grupos sociales distintos, la clase social ha pedido poder explicativo. Sin embargo, los datos que aportan los investigadores no son del todo concluyentes.

Así, por ejemplo, el trabajo de Tomlinson (2003) plantea con el propio título Lifestyle and social class que las diferencias de consumo y los distintos patrones alimentarios, son resultado del capital social de los individuos. Siguiendo a Bourdieu y en base a las encuestas oficiales de salud y estilos de vida de diferentes décadas británicas, concluye que la clase social y el género siguen marcando importantes diferencias en el consumo alimentario en las sociedades modernas, como es el caso que el autor analiza en la sociedad británica. La investigación cuantitativa, en este caso, confirma la pervivencia de diferencias sociales en el consumo de alimentos en función del género y el nivel educativo en la sociedad. Se apoya en las teorías de Bourdieu para desarrollar estas conclusiones. También Cheng et al. en 2007 confirman que las diferencias sociales en las prácticas alimentarias no han desaparecido cuando lo que se analiza es el uso del tiempo dedicado a la alimenta- 
ción, aunque han ganado importancia nuevas formas de división social. Lo que hoy marca las diferencias en las formas de alimentarse está más ligado al capital cultural y la composición de los hogares que a otras formas de estratificación social, como la participación en el mercado de trabajo.

\subsection{Los estudios comparados y la homogeneización alimentaria}

El debate en torno a la dualidad entre una alimentación culturalmente asentada o unos hábitos más homogéneos como resultados de la modernidad social sólo puede ser resuelto a través de la comparación entre sociedades. De los estudios comparados, caben destacar los resultados de tres investigaciones: el trabajo que Warde et al. realizan en 2007 que estudia el tiempo dedicado a la alimentación en Francia, Reino Unido, Estados Unidos, Noruega y Países Bajos, a través de las encuestas de uso del tiempo; el estudio realizado con las encuestas de gasto de Díaz-Méndez y GarcíaEspejo (2012) comparando la sociedad española y británica, y el estudio también de gasto realizado por López Martín-Lagos (2011). Es este un debate que sólo puede ser resuelto con estudios comparados que ponga en relación variables culturales y sociales en contextos socioeconómicos distintos, los trabajos existentes tienen varios aspectos en común: por un lado, se trata de estudios de carácter cuantitativo cuyos datos se obtienen bien de las encuestas de gasto bien de las de tiempo. Esta orientación metodológica propicia una visión más homogénea de la realidad alimentaria, pues analiza comportamientos cuantificables y minimiza los efectos de aspectos generadores de mayor diversidad y con un más alto anclaje cultural (creencias y valores, por ejemplo). Sin embargo, y a pesar de esta visión cuantitativa del cambio, ambos estudios confirman tanto similitudes como diferencias entre países. Las semejanzas son más atribuibles a variables de tipo social, pero resulta complicado determinar si las diferencias se deben o no al contexto sociocultural. En este sentido el trabajo de López Martín-Lagos (2011) que analizan los 27 países de la Unión Europea confirma la denominada Ley de Engel en la que el PIB está en relación inversa al gasto que los hogares destinan a la alimentación.

Los tres estudios confirman un cierto proceso de convergencia en Europa. Se puede desprender de ellos que hay tendencias comunes, como la mayor implicación de los hombres en la alimentación doméstica o la reducción del tiempo y el gasto en alimentación. Detrás de ellas también fuerzas unificadoras, como la persistencia de la comida familiar en el hogar. Pero también persisten las diferencias y se detectan más en la alimentación extradoméstica, tanto en términos de tiempo como de gasto.

\subsection{Desaparición o resistencia de la comida familia}

En relación a la pervivencia de la comensalidad familiar los trabajos cuantitativos y los cualitativos ofrecen resultados dispares. Podríamos afirmar que en los trabajos sobre estos temas coinciden al constatar la preocupación por el cambio tanto como 
la lentitud con la que sucede. No hay datos concluyentes al respecto, pero son muchos los autores que lo plantean como eje de sus trabajos. Para Warde et al. (2007) las prácticas de la comida familiar siguen presentes a lo largo del tiempo y lo confirman también otros autores al analizar grupos sociales previsiblemente más proclives a la inestabilidad alimentaria, como la población adolescente, (Gallegos et al., 2011). Las comidas se preparan y comen en familia, aunque no todos los adolescentes australianos del estudio valoren por igual este hecho.

La diferencia en la valoración y en las prácticas también se pone de manifiesto entre la población rural y urbana. Lupton, en el año 2000, bajo el título The heart of the meal, y a través de entrevistas en profundidad a 34 parejas australianas, analiza las comidas realizadas y las creencias asociadas al comer. Los resultados indican que la población analizada tiene una creencia compartida acerca de la comida familiar y la sigue, aunque con variaciones en algunos platos nuevos. La comida familiar preparada y realizada en el hogar, se asocia a comida sana y adecuada. Se confirma el conservadurismo y el mantenimiento de la tradición y siguiendo a Bourdieu la autora confirma la relación entre la clase social y los hábitos alimentarios.

Otros autores, por el contrario, ponen más énfasis en la disminución de la comida familiar y el crecimiento de la individualización de las prácticas alimentarias. En Bélgica, Mestdag y Glorieux (2009), a través de estudios longitudinales con las encuestas de uso del tiempo, analizan los cambios en la comida familiar y detectan transformaciones que apoyarían un cambio en las comidas en grupo, pero no un drástico abandono de esta práctica. Concretan que comer es una actividad social que se comparte con los miembros del hogar y que las variaciones están en relación directa al cambio en las estructura de los hogares. Invitan a un análisis cualitativo más a fondo para precisar la naturaleza de estos cambios.

Sin embargo, datos posteriores y a través de un análisis multi-nivel realizado por Davidson y Gauthier (2010) con datos del informe Programa de Evaluación Internacional de Alumnos (PISA) y la World Values Survey (WVS) no concuerdan con esta predicción. Estas autoras analizan los datos de 43 países para determinar los factores que predicen la realización de la comida familiar. Ni la renta, ni el tamaño de la familia explican esta conducta; es una mayor interacción familiar y hogares más amplios los más propensos a la comensalidad familiar. El estudio, al ser comparado, permite confirmar que los valores nacionales ayudan a explicar las variaciones internacionales, aunque también se ve que las familias reservan tiempo para compartir la comida familiar, sea cual sea el país de referencia.

El análisis del cambio alimentario encuentra un foco de estudio privilegiado en las transformaciones de la alimentación entre la población inmigrante. El choque cultural altera las pautas de consumo alimentario de la población que llega a un nuevo lugar y se analiza si estas pautas se mantienen o se abandonan con el fin de comprender el proceso de aculturación y/o asimilación (Cleveland et al., 2009). Los estudios confirman el mantenimiento de la identidad étnica a pesar de la inmersión en la sociedad receptora. El ejemplo presentado por los autores, de libaneses en Canadá, refleja una importante interacción entre las personas y no se da ni un 
proceso de aculturación, ni de inmersión, ni de resistencia. Es un proceso complejo en el que el inmigrante se mueve entre la adquisición de las características de la cultura dominante pero manteniendo fuertes lazos con su cultura de origen.

Por ello se estudia la transformación que sufren los hábitos alimentarios con el cambio de lugar y la comida como factor generador de identidad. Para unos la clave del cambio está ligada al origen y a la forma en que los individuos arrastran sus patrones alimentarios al lugar al que se trasladan. Es muy interesante el trabajo de Gvion (2006) sobre la comida de los palestinos en Israel al mostrar la conexión entre los discursos políticos, los discursos nacionales y los discursos alimentarios. Muestran el potencial de la alimentación para reforzar y mantener la identidad cultural cuando ésta se pone en peligro en un contexto culturalmente adverso. Sin embargo, también los estudios en este campo han precisado que las respuestas de cambio en la dieta que se detectan en las poblaciones inmigrantes no son necesariamente resultado del choque cultural, sino que en ellos intervienen también las limitaciones económicas del inmigrante. En el trabajo con el título We eat meat every day (Guarnaccia et al., 2012) se muestra que la transformación que se produce no es resultado de una falta de conocimientos sobre la dieta, sino de las dificultades económicas para componer una dieta saludable que se agravan al no disponer de productos conocidos.

En cualquier caso, la literatura analizada pone de manifiesto las discrepancias acerca de la continuidad de los patrones culturales alimentarios. La cultura alimentaria sirve de soporte de actuación para decidir qué y cómo comer, qué es apropiado e inapropiado hacer. Además, las dinámicas de las sociedades modernas son el contexto en el que estas prácticas alimentarias se desenvuelven e introducen cambios en ellas. Sin embargo, todo hace pensar que la comida tradicional no ha desaparecido dando paso a una nueva forma de comer radicalmente distinta, pero también todos los analistas coinciden en constatar que se están produciendo cambios que es necesario explorar para comprender la dimensión y las consecuencias de esta transformación. Ante esta ambigüedad, no es extraño que los analistas se hayan introducido en uno de los debates teóricos clásicos de la Sociología, la pervivencia de las clases sociales.

\section{Identidades alimentarias: alimentación, cuerpo y salud}

Si el estudio de los hogares ha derivado hacia la desigualdad alimentaria, el estudio de los sujetos ha llevado al análisis de las dietas. En éste confluyen tres áreas que muestran la multidimensionalidad de la alimentación a la vez que reflejan las dificultades de análisis: la alimentación, la salud y el cuerpo. Se muestra con ello uno de los efectos más individuales de la globalización alimentaria, el proceso de individualización de las elecciones alimentarias. La modernidad alimentaria se refleja en la confusión del comensal a la hora de decidir qué comer y cómo elegir 
los alimentos. Es este un asunto ligado a la distancia entre consumidor y productor, pero también al descenso de pautas alimentarias culturales sobre las que afianzar las elecciones apropiadas y a un contexto alimentario donde la diversidad hace más complicada la selección de lo que es bueno para comer. No es extraño, por tanto, que se detecte confusión y ansiedad entre el comensal y que sea la salud uno de los aspectos más preocupantes en tanto que es el reflejo más claro de una elección correcta o incorrecta.

La salud se presenta como un valor que marca las elecciones alimentarias de los comensales y también muestra diferencias relevantes entre los individuos en función de variables de tipo social. Aunque esta temática es una constante desde finales de la década de los noventa, y ha derivado hacia diferentes áreas, tiene elementos vertebradores. En primer lugar, los estudios se apoyan prioritariamente en metodologías cualitativas, poniendo de manifiesto tanto la dificultad de estudio como la intención de abordarlo de forma abierta. Estas aproximaciones permiten desentrañar los aspectos menos explícitos de la alimentación y ofrecen una imagen muy rica de las practicas alimentarías a través de los significados que le atribuyen los sujetos de estudio. En segundo lugar, los trabajos sitúan a las mujeres en el punto de mira de las dificultades para comer correctamente, bien como responsables principales y/o únicas de la alimentación, bien como diana sobre la que recaen las presiones sociales que obligan a mantener unas pautas alimentarias socialmente apropiadas.

En general, los trabajos sobre salud, alimentación y cuerpo reflejan la confusión del comensal. Rodeado de alimentos y saturado de información, el comensal moderno se muestra confuso en sus elecciones. Ambos rasgos, desconfianza y confusión, afectan a las elecciones alimentarias y son los elementos más definidores de la modernidad alimentaria.

\subsection{Problemas con el cuerpo: género y dieta}

El trabajo de Lupton en 1994 abre el campo de estudio de la alimentación y el cuerpo. En su trabajo Food, memory and meaning. The symbolic and social nature of food events emplea una técnica que denomina memory-work; una metodología cualitativa innovadora en la que se pide a estudiantes universitarios rememorar sus recuerdos de infancia. A través de estos relatos destapa el papel de la alimentación en la creación de relaciones sociales y el significado de la comida. Destaca en particular el interés que tienen las orientaciones sobre la salud en la alimentación y explora los factores que explican su trasgresión y su seguimiento acudiendo a los significados que los comensales atribuyen a la alimentación.

Germov y Williams, autores pioneros en la constitución de una Sociología de la Alimentación a partir de su libro A sociology of food and nutrition. The social appetite (2008) presentan en 1996 un trabajo titulado The sexual division of dieting. Women's voices en el que exploran, con grupos de discusión, los discursos de las mujeres australianas que están a dieta. Muestran la forma en que el género influye 
en las decisiones alimentarias y cómo el ideal de delgadez dominante es recreado por las propias mujeres para justificar sus prácticas alimentarias.

Regnier y Massullo (2009) en su trabajo Obesity, taste and consumption entrevistan a un importante número de mujeres francesas para explorar cómo se pliegan o reaccionan ante las normas. Plantean la diferencia de discursos en función de la clase social y constatan que las clases privilegiadas tienen integrados en sus discursos la norma de salud, como un comportamiento ascético, mientras que las clases populares elaboran respuestas más ligadas al gusto. Las limitaciones económicas, no obstante, no son las únicas restricciones que diferencian las orientaciones alimentarias entre las mujeres de diferentes clases sociales.

Algunos trabajos estudian por qué las mujeres no logran seguir las dietas contra el sobrepeso y la obesidad. Warin et al. (2008) analizan los discursos alimentarios de las madres en Australia para dejar en evidencia la relación entre la construcción de la maternidad y la obesidad. También en España, Martín Criado (2010) analiza la dificultad de las mujeres de clase baja para seguir una dieta. A partir de grupos de discusión y entrevistas muestra cómo la dificultad para hacer dieta está directamente relacionada con los papeles tradicionales de madres que impiden a las mujeres ocuparse de sí mismas. Se encuentra un resultado similar en mujeres marroquies obesas (Batnizky, 2008). Los roles domésticos en una sociedad con una fuertes división de género en el hogar están íntimamente relacionados con la salud de las mujeres e interviene en su mayor propensión a la obesidad.

Las cuestiones que vinculan alimentación y cuerpo son desarrolladas a través del análisis de la imagen corporal. Spurgas (2005) entrevista a mujeres universitarias de distintas razas para conocer la imagen que tienen de sus cuerpos y los factores que conforman estas imágenes. Los estudios que relacionan raza y cuerpo no parecen confirmar la relación entre estas variables y tanto las mujeres blancas como las de raza negra actúan de modo similar. Se ha demostrado, por ejemplo, a través de estudios cuantitativos, que las jóvenes adolescentes tienen una percepción similar de su peso y los factores psicosociales como la depresión o la inseguridad les afectan por igual en el desarrollo de conductas alimentarias patológicas (Haff, 2009). Pero la presión social que incide en las mujeres está presente en estos trabajos, como se ve también en la relación entre práctica deportiva y trastornos alimentarios. Crissey y Honea (2006), por ejemplo, explican la mayor propensión a cuidar el peso y actuar para reducirlo entre las jóvenes que practican deportes femeninos.

Las temáticas sobre el cuerpo se introducen en un tema tradicionalmente tratado por médicos y psicólogos: la anorexia. Fox et al. (2005) analizan las redes que animan a seguir estas prácticas alimentarias a quienes las sufren. Y exploran las páginas web denominadas en la jerga ProAna. Estos trabajos continúan con una orientación más centrada en los aspectos sociales que condicionan estas conductas, como los análisis realizados en mujeres jóvenes anoréxicas internadas en un centro británico (Rich, 2006) o la confirmación de la relación entre la clase social media y alta y la anorexia, mostrada a través de entrevistas a mujeres francesas por Darmon (2009). 
En general estos trabajos se sustentan en orientaciones teóricas que exploran cómo se construye la identidad a través del cuerpo y tienen un marcado carácter psicosocial. Analizan la relación entre los desordenes alimentarios y las crisis de identidad que se producen en la adolescencia y explican los trastornos como reflejo de una autoimagen corporal negativa que busca soluciones a los conflictos de identidad a través de comportamientos alimentarios específicos. Estos trastornos son, como indican los analistas (Matthews et al. 2012), propios de personas insatisfechas con su vida y su imagen corporal. El elemento teórico común de estos trabajos relacionados con la salud y el género es su gran afinidad con las teorías de Bourdieu. Relacionan las elecciones con las formas en que se forja el gusto y la identidad a través de las prácticas alimentarias aprendidas en los grupos sociales de pertenencia.

\section{Conclusiones}

Algunos autores han justificado, desde diferentes posiciones, la existencia de un cuerpo científico con entidad propia dedicado al estudio sociológico de la alimentación. Esto ha cristalizado no solamente en libros dedicados a esta temática, sino en la incorporación en algunos manuales de Sociología General de capítulos dedicados a este campo de estudio (Macionis y Plummer, 2004). La perspectiva adoptada en este trabajo tiene también por objetivo averiguar si estamos ante un campo de estudio específico y lo hacemos a través del repaso de las publicaciones científicas que la Sociología internacional dedica a la alimentación. A la vista de los datos se pueden considerar algunas cuestiones de interés para tomar una decisión al respecto.

La evolución temática seguida por estos trabajos y el aumento creciente de artículos permite confirmar que la Sociología ha formulado y comprobado de manera sistemática hipótesis acerca de los aspectos sociales de la alimentación contemporánea. Se ha adaptado analizando las trasformaciones propias de la modernidad y, si bien ha iniciado su andadura con la exploración de los cambios en el sector agrario, ha derivado hacia el análisis de las problemáticas de un sistema agroalimentario de creciente complejidad y en el que no solo hay productores, sino también distribuidores, consumidores, comensales e instituciones. Los estudios desarrollados han puesto el acento en los efectos de la globalización alimentaria y la expansión del mercado mundial de alimentos. Se detecta una adecuación a las circunstancias cambiantes de las sociedades actuales y es claro el esfuerzo por comprender las dimensiones de estas transformaciones y de sus efectos sobre las sociedades y los individuos.

Al igual que sucede en otras disciplinas, el centro de atención de la Sociología hacia la alimentación se ha orientado, preferentemente, hacia las sociedades de sobreabundancia alimentaria, en las que viven y trabajan los investigadores, de ahí que los estudios tengan un cierto sesgo etnocentrista en sus temáticas. Sin embargo, el haber iniciado su andadura a través de la Sociología Rural, las problemáticas de los países en desarrollo han ocupado los primeros años, con una orientación más 
centrada en la producción y menos en el efecto de las transformaciones sobre el ámbito no productivo. La Sociología del Consumo también ha contribuido al empuje de los estudios de los últimos años, aunque ambas perspectivas han contado con escasa conexión. Esto no ha impedido que los investigadores hayan analizado a fondo los factores de cambio, el carácter desestabilizador de las transformaciones, el papel de las instituciones como generadoras de relaciones de desigualdad y poder o el desequilibrio presente en sociedades de aparente estabilidad alimentaria.

Los trabajos tienen una clara orientación empírica y asumen un reducido número de teorías. La mayoría de los estudios tienen, además, una orientación inductiva; pasan de lo particular a lo general formulando hipótesis a partir de la observación y los datos. El resultado, más que teorías que logren continuidad, es un cúmulo de casos de estudio.

La metodología adoptada también aporta una explicación sobre este abordaje sociológico. Los trabajos que persiguen conocer los discursos que explican las acciones (sobre la tierra, sobre la compra, sobre la dieta, sobre los otros, ....) se apoyan tanto en el objeto como en el método; de hecho, la frecuente elección de la metodología cualitativa ha facilitado una orientación abierta ante la ausencia de una teoría que hubiera podido ayudar a entender y a analizar. Los trabajos cuantitativos contribuyen de otro modo a la creación de un cuerpo disciplinario propio. Plantean hipótesis precisas con el fin de alcanzar objetivos, y para ello se ven en la necesidad de operativizar la "globalización alimentaria", de ofrecer alguna de las múltiples dimensiones del concepto para su exploración.

Merece una atención especial la escasez de estudios comparados, pues con frecuencia los autores finalizan sus artículos recordando la necesidad de reproducir el estudio en otros contextos con el fin de testar su grado de generalización. Pero además, la comida ha tenido históricamente un fuerte anclaje cultural y solo los estudios históricos y comparados podrían decir si los cambios alimentarios trascienden el momento y el lugar. Además, las hipótesis derivadas de la estandarización de comportamientos alimentarios, como resultado de la globalización, requieren de comparaciones que lo verifiquen o lo desmientan para ver si el ritmo y las dimensiones de los cambios son o no coincidentes.

Los estudios que hemos revisado coinciden al intentar comprender los efectos provocados por la globalización alimentaria sobre el sistema agroalimentario. La evolución de la literatura sociológica sobre alimentación y su intensificación en los inicios del siglo XXI es un reflejo del rápido desarrollo del sector agroalimentario, pero refleja también su creciente problematización. Nuevas formas de organización productiva, nuevas formas de comercialización, nuevos agentes en la cadena agroalimentaria, nuevos valores de consumo, son parte de un mismo fenómeno: una producción intensiva de alimentos para un consumo alimentario de masas. Los analistas exploran estas nuevas situaciones en relación con la globalización alimentaria y vinculan su problematización a la creciente distancia entre productores y consumidores.

Los análisis sociológicos sobre la alimentación que exploran la situación de los países pobres coinciden al afrontar las trasformaciones de sociedades que sufren la 
intensificación de una actividad agraria de carácter tradicional. La peculiaridad de estos estudios, frente a los estudios clásicos de la Sociología Rural, es que no se detienen en el análisis de los aspectos productivos, sino que afronta los efectos de las transformaciones agrícolas sobre la alimentación de los ciudadanos, en particular de sus dietas. Comienzan estudiando la desnutrición, siguen con la malnutrición, y finalizan analizando el sobrepeso y la obesidad como consecuencia del mismo fenómeno: la integración de la agricultura en el proceso de industrialización y de los alimentos en los mercados internacionales. En definitiva, narran el impacto de la globalización económica sobre los más pobres; la mayoría de los autores explican estos efectos sobre la producción y algunos lo hacen sobre la dieta.

En otros países se exploran también las consecuencias sobre las formas tradicionales de organizar la cadena agroalimentaria, propia de los pequeños productores, aunque en este caso se discute de manera más intensa acerca del proceso de convencionalización de la agricultura tradicional. Su estudio se ha desarrollado ampliamente en Europa, Australia y América del Norte, en menor medida en países latinoamericanos, y es casi inexistente la literatura en torno a la situación de los países africanos o asiáticos.

Para unos, las formas alternativas de producción resisten el impacto del cambio en el sistema agroalimentario bajo la lógica de ofrecer confianza y seguridad al consumidor a través de nuevas formas de comercialización (canales alternativos, producciones locales); para otros, no se sobrevive al impacto de la globalización alimentaria y es sólo cuestión de tiempo que toda la agricultura se adapte a un consumo alimentario de masas, cuya oferta estará dominada por las grandes corporaciones alimentarias. En medio de este debate se analiza el papel de los agentes de la cadena, la pérdida de poder del productor, el cambio de valores del consumidor, el aumento de la relevancia de los distribuidores y las industrias agroalimentarias o el papel regulador de las instituciones públicas (los Estados) y privadas (los grupos de acción).

Los investigadores estudian, en paralelo pero sin confluencias, el mundo productivo y el mundo del consumo. El análisis del mundo productivo pone en evidencia la disminución de la capacidad de control de los productores a favor de otros agentes. Los estudiosos del campo del consumo muestran la preocupación de los consumidores ante lo riesgos alimentarios. Algunos autores logran vincular ambos campos y aunque se sigue trabajando en el proceso de inserción de la agricultura en las lógicas del mercado global, ésta se relaciona con la comercialización de los productos y con las demandas de los consumidores. Además, aparecen nuevos valores que movilizan nuevas formas de producción con el fin de asegurar alimentos más sanos y respetuosos con el entorno.

Todos coinciden al analizar los efectos de la globalización sobre la alimentación, por lo que no cabe duda de que están claramente anclados en los problemas sociológicos actuales. Algunos de ellos presentan solamente resultados de carácter económico. Otros introducen una dimensión política al considerar la interacción entre los actores y el poder que ejercen en el sistema agroalimentario. Es habitual encon- 
trar estudios en los que se plantea, desde la perspectiva del conflicto, una orientación crítica con los roles de los distintos agentes de la cadena agroalimentaria.

El desarrollo de la teoría del actor red (ANT) aplicada al análisis del cambio en la cadena agroalimentaria es el soporte teórico más elaborado. Se busca poner en evidencia el efecto del poder sobre los grupos más débiles y vulnerables de la sociedad. Esta perspectiva vincula sujetos y objetos (naturaleza y sociedad) y con ello permite ver el modo en que los actores de la cadena redefinen y transforman el alimento. Frente a las teorías clásicas de la acción racional, esta orientación va más allá de la explicación del cambio basada en la atribución de sentido a la acción. No se pretende solamente comprender las motivaciones de los consumidores hacia los productos alimentarios, sino también cómo los actores le dan (o le quitan) valor a lo largo de la cadena. En algunos casos se comprenden mejor los procesos y no solo los resultados, pero son pocos los que además de estas dimensiones, económica y política, incorporan la dimensión cultural al análisis.

Los debates sobre los efectos de la globalización son duales: por un lado, quienes afirman que la globalización llevará a la desaparición de las formas alternativas de producción; por otro lado, los que apuestan por una fragmentación de alternativas que conviven, con mayor o menor grado de éxito. Los estudios comparados ayudan a matizar la dualidad, pues en la medida en que los cambios se analizan en relación al contexto en el que se producen, las explicaciones sobre sus efectos son más amplias y abren opciones para comprender cómo afectan otras fuerzas (no solo las económicas). También deberían permitir conocer si la orientación y la intensidad de los cambios son similares en contextos distintos, aunque la escasez de trabajos no ayuda a clarificar con total satisfacción este aspecto.

A pesar de los intentos por unir producción y consumo el análisis "agroalimentario" se separa del análisis de los "hábitos alimentarios”. El cambio dentro de los hogares es efecto del mismo proceso, de la globalización alimentaria, pero no sigue la misma lógica de análisis, pues no se estudian en relación con los cambios en la producción, y en algunos casos tampoco como efecto de la mercantilización de los alimentos. Son estudios heterogéneos, quizás por no compartir un marco teórico básico de análisis como sucede a los anteriores, aunque se pueden identificar orientaciones teóricas clásicas.

Los trabajos de Bourdieu son el soporte de muchos estudios que exploran las desigualdades y el cambio alimentario en el ámbito del consumo. Las teorías de la distinción de Bourdieu se confrontan con las propuestas sobre la onmivorosidad y la hibridación. Se argumenta si las elecciones alimentarias son una respuesta para mantener un status de diferenciación social que hace a ciertos grupos sociales adoptar hábitos que les diferencian de otros grupos sociales peor situados. Otras explicaciones se sustentan en la idea de que las fronteras culturales son cada vez más permeables y que es precisamente la erosión progresiva de las diferencias lo que hace que ciertos comportamientos alimentarios sean reflejo de la onmivorosidad cultural, es decir, de pautas alimentarias de apertura hacia formas de comer nuevas y propias de culturas diversas. En general, los estudios sobre las desigualdades sociales atraviesan los análisis del ámbito doméstico: desigualdades de salud, de 
renta, de género, de raza, de lugar de residencia. Coinciden al mostrar el acceso desigual a los recursos y los efectos de la globalización alimentaria sobre los individuos más vulnerables.

La preocupación por el grado de estandarización de la alimentación y la pérdida cultural se focaliza en el estudio sobre la resistencia o la desaparición de la comida familiar. Esta dicotomía se asemeja a la resistencia o desaparición del productor alternativo seguida en los debates sobre la producción agraria, pero aquí se aplica al mantenimiento de los hábitos alimentarios y a la capacidad de resistencia de las culturas locales frente a los modelos alimentarios dominantes. Estos modelos difunden y potencian formas más homogéneas de actuación, propician elecciones alimentarias similares y con ello hacen descender las pautas alimentarias culturalmente diferenciadas. Se adoptan posiciones excesivamente dicotómicas, pues para unos la cultura global produce integración y para otros desintegra; y son escasos los trabajos que plantean que la globalización pueda generar fuerzas tanto unificadoras como fragmentadoras.

Los analistas han logrado unir producción y mercantilización en sus estudios, pero ambos campos se han mantenido al margen del ámbito no productivo de la alimentación: el hogar. Por ello es fácil diferenciar dos áreas de análisis: el mundo productivo y el mundo doméstico, ambos separados (o unidos) por el comprador. Los efectos de la globalización alimentaria se estudian en ambos, y se comparte la preocupación acerca de la homogeneización de comportamientos alimentarios. El comensal, y con él también la persona que transforma en el hogar el alimento, quedan fuera del análisis del ámbito no doméstico Sus decisiones parecen reducidas y asimiladas al rol de comprador: a sus actitudes, a sus preferencias, a sus valores; y se sobreentiende, erróneamente, que éstas son las mismas que las del comensal y las del cocinero.

Los estudios ligados al ámbito doméstico tienen un desarrollo paralelo al resto, pero no confluyen, y sus problemáticas se mantienen aisladas. Por un lado, la internacionalización de los alimentos en los mercados globales altera la producción, la distribución y la compra, por ello se estudia la resistencia o la desaparición de las formas alternativas de producir y de vender y el efecto que sobre ello tiene el cambio de valores de los consumidores. Los cambios alimentarios se ejemplifican a través de la demanda y la oferta de productos orgánicos. Por otro lado, la interconexión de las sociedades y la permeabilidad de las fronteras afectan a la forma en que se mantienen o se deterioran las prácticas alimentarias asentadas culturalmente. En particular, la desaparición o resistencia de la comida familiar. Pero en esencia, aún siendo dos áreas estudiadas por separado, en ambos casos se analizan como resultado del proceso de globalización alimentaria. Este es precisamente el marco común de análisis sobre el que se asientan los estudios de lo que deberíamos comenzar a llamar una Sociología de la Alimentación.

Más allá de los efectos de cambio alimentario, lo que se observa es el análisis de un proceso, la globalización alimentaria, que produce un entorno general de incertidumbre sobre el que se asientan las decisiones de los individuos. Decisiones sobre 
qué producir, qué vender, qué comprar, qué comer o qué tirar, pero también cómo y por qué hacerlo.

La incertidumbre da lugar a comportamientos contradictorios: la fractura y la homogeneidad, la estandarización y la diversidad, son resultado de un mismo fenómeno. Así, aunque los analistas muestran los efectos de la globalización alimentaria, lo que vemos al mirarlo conjuntamente, es el entorno de trasformación sobre el que se están produciendo las complejas y diversas acciones humanas relacionadas con el alimento.

En definitiva, se emplean teorías y métodos de otros campos sociológicos para comprender la alimentación, al igual que sucede en otras muchas áreas de estudio. Pero no se trata tanto de orientaciones "prestadas" como de aplicaciones a un objeto de estudio que requiere de todo el instrumental sociológico para su comprensión. Aunque esta orientaciones tengan vías de mejora, tanto teórica como metodológicamente, se puede afirmar que en el estudio que realiza la Sociología sobre la alimentación se está produciendo un cuerpo teórico que sirve de orientación para la investigación futura, pues se cuenta con un soporte común de análisis que enmarca todos los trabajos en el campo teórico de la globalización alimentaria.

No se puede concluir este trabajo sin recordar las limitaciones de los registros utilizados: se trata de artículos en revistas de Sociología principalmente de lengua inglesa. Quedan fuera aquellos trabajos de otras lenguas y de otras disciplinas que complementarían, sin duda, lo que se ha visto en este trabajo. Cabe destacar, en particular, que no se han estudiado aquí las revistas de Antropología, disciplina cuyas fronteras con la Sociología, y más en los análisis de la alimentación, resultan muy difusas. Tampoco se han explorado los trabajos de las ciencias no sociales que cuentan con una amplia producción científica: las Ciencias de la Salud, en particular los estudios nutricionales y epidemiológicos, la Bioquímica o la Ingeniería Agrónoma, son tres disciplinas sin las cuales los estudios sociales no quedan completos.

Concluyendo, se puede decir que este recorrido por los textos sociológicos sobre la alimentación nos permite hablar de la Sociología de la Alimentación como la ciencia que analiza y explica los problemas derivados de la globalización alimentaria. La aportación más significativa de esta nueva disciplina científica es su capacidad para analizar los vínculos entre estructuras y acciones, entre objetos y sujetos, en definitiva, entre naturaleza y sociedad. Esto es posible gracias a la gran potencialidad del alimento y la alimentación para capturar la complejidad y la multidimensionalidad del mundo social. 


\section{Bibliografía}

Alkon, A. H. y Norgaard, K. M. (2009) "Breaking the Food Chains: An Investigation of Food Justice Activism” Sociological Inquiry, 79: 289-305.

Batnitzky, A. (2008) "Obesity and household roles: gender and social class in Morocco" Sociology of Health \& Illness, 30: 445-462.

Beardsworth, A. y Keil, T. (1997) Sociology on the menu. An invitation to the study of food and society, London y New York: Routledge .

Berg, L.; Kjaernes, U.; Ganskau. E.; Minina, V.; Voltchkova, L.; Halkier, B. y Holm, L. (2005) "Trust in food safety in Russia, Denmark and Norway" European Societies, 7: 103-129.

Bonanno, A.; Constance, D. y Hendrickson, M. (1995) "Global Agrofood corporations and the state-the Ferruzzi case" Rural Sociology, 60: 274-296.

Bonanno, A. y Constance, D. (2006) "Corporations and the state in the global era: The case of seaboard farms and Texas" Rural Sociology, 71: 59-84.

Brunori, G.; Rossi, A. y Guidi, F. (2012) "On the New Social Relations around and beyond Food. Analyzing Consumers' Role and Action in Gruppi di Acquisto Solidale (Solidarity Purchasing Groups)" Sociologia Ruralis, 52: 1-30.

Buck, D.; Getz, C. y Guthman, J. (1997) "From farm to table: The organic vegetable commodity chain of northern California” Sociologia Ruralis, 37: 3-20.

Campbell, D. J. (1999) "Response to drought among farmers and herders in southern Kajiado District, Kenya: A comparison of 1972-1976 and 1994-1995” $\mathrm{Hu}$ man Ecology, 27: 377-416.

Campbell, H. y Liepins, R. (2001) "Naming organics: Understanding organics standards in New Zealand as a discursive field" Sociologia Ruralis, 41: 1-21.

Che, D.; Week, A. y Veeck, G. (2005) "Sustaining production and strengthening the agritourism product: Linkages among Michigan agritourism destinations” Agriculture and Human Values, 22: 225-234.

Cheng, S. L.; Olsen, W.; Southerton, D. y Warde, A. (2007): “The changing practice of eating: evidence from UK time diaries, 1975 and 2000" British Journal of Sociology, 58: 39-61.

Clarke, S.; Varshavskaya, L.; Alasheev, S. y Karelina, M. (2000) "The myth of the urban peasant” Work Employment and Society, 14: 481-499.

Cleveland, M.; Laroche, M.; Pons, F. y Kastoun, R. (2009) "Acculturation and consumption: Textures of cultural adaptation" International Journal of intercultural relations, 33: 196-212.

Crissey, S. R. y Honea, J. C. (2006) "The relationship between athletic participation and perceptions of body size and weight control in adolescent girls: The role of sport type” Sociology of Sport Journal, 23: 248-272.

Darmon, M. (2009) "The Fifth Element: Social Class and the Sociology of Anorexia” Sociology the Journal of the British Sociological Association, 43: 717-733.

Davidson, R. y Gauthier, A. H. (2010) “A cross-national multi-level study of family meals” International Journal of Comparative Sociology, 51: 349-365. 
Díaz-Méndez C. y García-Espejo, I. (2012) “Tendencias en la alimentación contemporánea: la homogeneización del gasto alimentario en España y Reino Unido" Revista Española de Investigaciones Sociológicas, 139: 21-43.

Deemer, D. R. y Lobao, L. M. (2011) "Public Concern with Farm-Animal Welfare: Religion, Politics, and Human Disadvantage in the Food Sector" Rural Sociology, 76: 167-196.

Dietz, T.; Frisch, A. S.; Kalof, L.; Stern, P. C. y Guagnano, G. A. (1995) "Values and vegetarianism-an exploratory analysis" Rural Sociology, 60: 533-542.

Dupuis E. M. (1993) "Sub-national state institutions and the organization of agricultural resource use - The case of the dairy Industry" Rural Sociology, 58: 440460.

Edin, K. (1991) "Surviving the welfare system - How AFDC recipients make ends meet in Chicago" Social Problems, 38: 462-474.

Fonte, M. (2008) "Knowledge, food and place. A way of producting, a way of knowing” Sociologia Ruralis, 49: 200-222.

Fox, N.; Ward, K. y O'Rourke, A. (2005) "Pro-anorexia, weight-loss drugs and the internet: an 'anti-recovery' explanatory model of anorexia" Sociology of Health Illness, 27: 944-971.

Gallegos, D.; Dziurawiec, S.; Fozdar, F. y Abernethie, L. (2011) “Adolescent experiences of 'family meals' in Australia” Journal of Sociology, 47: 234-260.

Garcia, H. y Lara, F. (2004) "Street food: urban informality and industrial modernity along Mexico's northern border" Sociologie du travail, 46: 42-53.

Germov, J. y Williams, L. (2008) A sociology of food and nutrition. The social appetite, Oxford: Oxford University Press.

Germov, J. y Williams, L. (1996) “The sexual division of dieting. Women's voices” Sociological Review, 44: 630-647.

Germov, J. y Williams, L. (ed.) (2000) A sociology of food and nutrition. The social appetite, Oxford University Press

Goodman, D. (1999) "Agro-food studies in the age of ecology. Nature, corporeality, bio-politics” Sociologia Ruralis, 39: 17-38.

Goodman, D. (2004) "Rural Europe redux? Reflections on alternative agrofood networks and paradigm change” Sociologia Ruralis, 44: 3-16.

Guarnaccia, P. J.; Vivar, T.; Bellows, A. C. y Alcaraz, G. V. (2012) "'We eat meat every day': ecology and economy of dietary change among Oaxacan migrants from Mexico to New Jersey” Ethnic and Racial Studies, 35: 104-119.

Guthman, J.; Morris, A. W. y Allen, P. (2006) "Squaring farm security and food security in two types of alternative food institutions" Rural Sociology, 71: 662684.

Gvion, L. (2006) "Cuisines of poverty as means of empowerment: Arab food in Israel” Agriculture and Human Values, 23: 299-312.

Haff, D. R. (2009) "Racial/Ethnic Differences in Weight Perceptions and Weight Control Behaviors among Adolescent Females” Youth \& Society, 41: 278-301.

Hall, A. y Mogyorody, V. (2001) "Organic farmers in Ontario: An examination of the conventionalization argument” Sociologia Ruralis, 41: 399. 
Heleski, C. R.; Mertig, A. G. y Zanella, A. J. (2006) "Stakeholder attitudes toward farm animal welfare" Anthorzoos, 4: 290-307.

Hendrickson, D.; Smith, C. y Eikenberry, N. (2006) " Fruit and vegetable access in four low-income food deserts communities in Minnesota” Agriculture and $\mathrm{Hu}$ man Value, 23: 371-383.

Holden, S. J.; Coppock, D. L. y Assefa, M. ( 1991) "Pastoral Dairy Marketing and household wealth interactions and their implications for calves and humans" Human Ecology, 19: 35-59.

Holm, L. y Halkier, B. (2009) "EU Food Safety Policy: Localizing contested governance” European Societies, 11: 473-493.

Hyers, L. L. (2006) "Myths used to legitimize the exploitation of animals: An application of Social Dominance Theory" Anthrozoos, 19: 194-210.

Inwood, S. M.; Sharp, J. S.; Moore, R. H. y Stinner, D. H. (2009) "Restaurants, chefs and local foods: insights drawn from application of a diffusion of innovation framework" Agriculture and Human Values, 26: 177-191.

Jehlicka, P.; Kostelecky, T. y Smith, J. (2013) "Food Self-Provisioning in Czechia: Beyond Coping Strategy of the Poor: A Response to Alber and Kohler's 'Informal Food Production in the Enlarged European Union' (2008)” Social Indicators Research, 111: 219-234.

Johnson, J. D. y Griffith, D. C. (1996) "Pollution, food safety, and the distribution of knowledge" Human Ecology, 24: 87-108.

Kalof, L.; Dietz, T.; Stern, P. C. y Guagnano, G. A. (1999) "Social psychological and structural influences on vegetarian beliefs" Rural Sociology, 64: 500-511.

Kenney, C. T. (2008) "Father doesn't know best? Parents' control of money and children's food insecurity” Journal of Marriage and Family, 70: 654-669.

Kobayashi, J. (2010) "Social Stratification and Food: Analyses of Effects on Health" Sociological Theory and Methods, 25: 81-93.

Koc, M.; Sumner, J. y Winson, A. (ed.) (2012) Critical perspectives in food studies, Oxford University Press.

Kortright, R. y Wakefield, S. (2011) "Edible backyards: a qualitative study of household food growing and its contributions to food security" Agriculture and Human Values, 28: 39-53.

Krueger, P. M.; Rogers, R. G.; Ridao-Cano, C. y Hummer, R. A. (2004) “To help or to harm? Food stamp receipt and mortality risk prior to the 1996 Welfare Reform Act” Social Forces, 82: 1573-1599.

Lockie, S.; Lyons, K.; Lawrence, G. y Mummery, K. (2002) "Eating green. Motivatins behind organic food consumption in Australia" Sociological Ruralis, 42: 23-40.

Lockie, S. y Halpin, D. (2005) “The 'conventionalisation' thesis reconsidered: Structural and ideological transformation of Australian organic agriculture” Sociologia Ruralis, 45: 284.

López Martín-Lagos, M. D. (2011) "Consumption and Modernization in the European Union” European Sociological Review, 27: 124-137. 
Lupton, D. (1994) "Food, memory and meaning-The symbolic and social nature of food events" Sociological Review, 42: 664-685.

Lupton, D. (2000) "The heart of the meal: food preferences and habits among rural Australian couples” Sociology of Health \& Illness, 22: 94-109.

Macionis, J. y Plummer, K. (2004) Sociología. Introducción a la sociología de la alimentación. De macro a micro, Madrid: Person Pentice Hall: 206-210

Marsden, T.; Banks, J. y Bristow, G. (2000) "Food supply chain approaches: Exploring their role in rural development” Sociologia Ruralis, 40: 424-438.

Martín Criado, E. (2010) "Las tallas grandes perjudican seriamente la salud: la frágil legitimidad de las prácticas de adelgazamiento entre las madres de clases populares" Revista Internacional de Sociología, 68: 349-373.

Matthews, M.; Zullig, K. J.; Ward, R. M.; Horn, T. y Huebner, E. S. (2012) “An Analysis of Specific Life Satisfaction Domains and Disordered Eating among College Students” Social Indicator Research, 107: 55-69.

McIntosh, A. (1996) Sociologies of food and nutrition, New York y London: Plenum Press.

Mennell, S.; Murcott, A. y van Otterloo, A. H. (1992) “The sociology of food: eating, diet, and culture" Current Sociology, $40, \mathrm{n}^{\circ} 2$, International Sociological Association. Sage.

Mestdag, I. y Glorieux, I. (2009) "Change and stability in commensality patterns: a comparative analysis of Belgian time-use data from 1966, 1999 and 2004” Sociological Review, 57: 703-726.

Miele, M. y Murdoch, J. (2002) "The Practical Aesthetics of Traditional Cuisines: Slow Food in Tuscany" Sociologia Ruralis, 42: 312-328.

Morton, L. W.; Bitto, E. A.; Oakland, M. J. y Sand, M. (2005) "Solving the problems of Iowa food deserts: Food insecurity and civic structure" Rural Sociology, 70: 94-112.

Murdoch, J. y Miele, M. (1999) "Back to nature. Changing "worlds of production" in the food sector" Sociologia Ruralis, 4: 465-483.

Nygard, B. y Storstad, O. (1998) "De-globalization of food markets? Consumer perceptions of safe food: The case of Norway" Sociologia Ruralis, 38: 35.

Pallotta, N. R. (2008) "Origin of adult animal rights lifestyle in childhood responsiveness to animal suffering” Society \& Animals, 16: 149-170.

Raynolds, L. T. (2002) "Consumer/producer links in Fair Trade coffee network" Sociologia Ruralis, 42: 404-424.

Regnier, F. y Masullo, A. (2009) "Obesity, taste and dietary consumption” Revue Française de Sociologie, 50: 747-773.

Rich, E. (2006) "Anorexic dis(connection): managing anorexia as an illness and an identity" Sociology of Health and Illness, 28: 284-305.

Roff, R. J. (2007) "Shopping for change? Neoliberalizing activism and the limits to eating non-GMO” Agriculture and Human Values, 24: 511-522.

Schafft, K. A.; Jensen, E. B. y Hinrichs, C. C. (2009) "Food Deserts and Overweight Schoolchildren: Evidence from Pennsylvania” Rural Sociology, 74: 153177. 
Shaw, A. (1999) "What are they doing to our food?: Public Concerns about Food in the UK” Sociological Research Online, 4: 258-271.

Schmeer K. K. (2005) "Married women's resource position and household food expenditures in Cebu, Philippines” Journal of Marriage and Family, 67: 399409.

Smith, C.; Butterfass, J. y Richards, R. (2010) “Environment influences food access and resulting shopping and dietary behaviors among homeless Minnesotans living in food deserts" Agriculture and Human Values, 27: 141-161.

Smith, P. A. y Smith, R. M. (1999) "Diets in transition: Hunter-gatherer to station diet and station diet to the self-select store diet” Human Ecology, 27: 115-133.

Sonderskov, K. M. y Daugbjerg, C. (2011) “The state and consumer confidence in eco-labeling: organic labeling in Denmark, Sweden, The United Kingdom and The United States" Agriculture and Human Values, 28: 507-517.

Spurgas, A. K. (2005) "Body image and cultural background” Sociological Inquiry, 75: 297-316.

Starr, A.; Card, A.; Benepe, C.; Auld, G.; Lamm, D.; Smith, K, y Wilken, K. ( 2003) "Sustaining local agriculture: Barriers and opportunities to direct marketing between farms and restaurants in Colorado" Agriculture and Human Values, 20: 301-321.

Tomlinson, M. (2003) "Lifestyle and social class" European Sociological Review, 19: 97-111.

Tomlinson, I. (2008) "Re-thinking the transformation of organics: The role of the UK government in shaping British organic food and farming” Sociologia Ruralis, 48: $133-151$.

Torres, R. (2002) "Linkages between tourism and agriculture in Mexico" Annals of Tourism Research, 30: 546-566.

Vinnari, M.; Rasanen, P. y Jokinen, P. (2013) "Attitudes towards Farm Animals as a Part of Belief Systems” Anthrozoos, 26: 111-123

Warde, A.; Cheng, S. L.; Olsen, W. y Southerton, D. (2007) "Changes in the practice of eating - A comparative analysis of time-use” Acta Sociologica, 50: 363385.

Warin, M.; Turner, K.; Moore, V. y Davies, M. (2008) "Bodies, mothers and identities: rethinking obesity and the BMI" Sociology of Health \& Illness, 30: 97-111.

Welch, J. R.; Ferreira, A. A.; Santos, R. V.; Gugelmin, S. A.; Werneck, G. y Coimbra C. E. A. (2009) "Nutrition Transition, Socioeconomic Differentiation, and Gender Among Adult Xavante Indians, Brazilian Amazon” Human Ecology, 37: 13-26.

Wimberley, D. W. y Bello, R. (1992): "Effects of foreign-investment, exports, and economic growth on third-world food consumption” Social Forces, 70: 895-921. 